\title{
Two-Dimensional Transition Metal Dichalcogenides: Synthesis, Biomedical Applications and Biosafety Evaluation
}

\author{
Xiaofei Zhou ${ }^{1 *}$, Hainan Sun ${ }^{2}$ and Xue Bai ${ }^{3}$ \\ ${ }^{1}$ Faculty of Science and Technology, Bohai Campus, Hebei Agricultural University, Cangzhou, China, ${ }^{2}$ Shandong Vocational \\ College of Light Industry, Zibo, China, ${ }^{3}$ School of Public Health, Shandong University, Jinan, China
}

\section{OPEN ACCESS}

Edited by:

Yin Liu,

Texas A\&M University, United States

Reviewed by:

Liwen Li,

Indiana University Bloomington

United States

Jie Liu,

South China Agricultural University,

China

*Correspondence:

Xiaofei Zhou

zhouxiaofeihappy@163.com

Specialty section:

This article was submitted to

Nanobiotechnology,

a section of the journal

Frontiers in Bioengineering and

Biotechnology

Received: 29 January 2020

Accepted: 06 March 2020

Published: 07 April 2020

Citation:

Zhou X, Sun H and Bai X (2020)

Two-Dimensional Transition Metal

Dichalcogenides: Synthesis,

Biomedical Applications and Biosafety

Evaluation.

Front. Bioeng. Biotechnol. 8:236.

doi: 10.3389/fbioe.2020.00236
Recently, two-dimensional transition metal dichalcogenides (2D TMDCs) have drawn certain attentions in many fields. The unique and diversified electronic structure and ultrathin sheet structure of 2D TMDCs offer opportunities for moving ahead of other 2D nanomaterials such as graphene and expanding the wide application of inorganic 2D nanomaterials in many fields. For a better understanding of 2D TMDCs, one needs to know methods for their synthesis and modification, as well as their potential applications and possible biological toxicity. Herein, we summarized the recent research progress of 2D TMDCs with particular focus on their biomedical applications and potential health risks. Firstly, two kinds of synthesis methods of 2D TMDCs, top-down and bottom-up, and methods for their surface functionalization are reviewed. Secondly, the applications of 2D TMDCs in the field of biomedicine, including drug loading, photothermal therapy, biological imaging and biosensor were summarized. After that, we presented the existing researches on biosafety evaluation of 2D TMDCs. At last, we discussed major research gap in current researches and challenges and coping strategies in future studies.

Keywords: 2D TMDCs, synthesis, modification methods, biomedical application, biosafety evaluation

\section{INTRODUCTION}

As the most well-known two-dimensional (2D) nanomaterials, graphene and graphene derivatives have been receiving great attention due to their fascinating physicochemical properties (Liu et al., $2012 b)$. In recent years, a newly emerging kind of $2 \mathrm{D}$ nanomaterial, two-dimensional transition metal dichalcogenides (2D TMDCs) got a lot of attention, whose generalized formula is $\mathrm{MX}_{2}$, where $\mathrm{M}$ represents transition metal and $\mathrm{X}$ represents chalcogen. $\mathrm{M}$ comprises of transition metals from the IVB to VIIB group, including Ti, V, Cr, Mn, Zr, Nb, Mo, Tc, Hf, Ta, W, and Re; X represents the chalcogenide elements, sulfur, selenium, and tellurium of the sulfur group (Figure 1). The metal coordination of 2D TMDCs is generally either trigonal prismatic or octahedral (Chhowalla et al., 2013). The synthetic method is gradually perfect and the present synthetic methods could be divided into two categories, top-down and bottom-up methods (Chen et al., 2015; Liu and Liu, 2018). In general, 2D TMDCs synthesized through top-down methods are mainly used in biomedical field, while 2D TMDCs prepared through bottom-up methods are mostly applied photoelectric devices and catalysis field. 


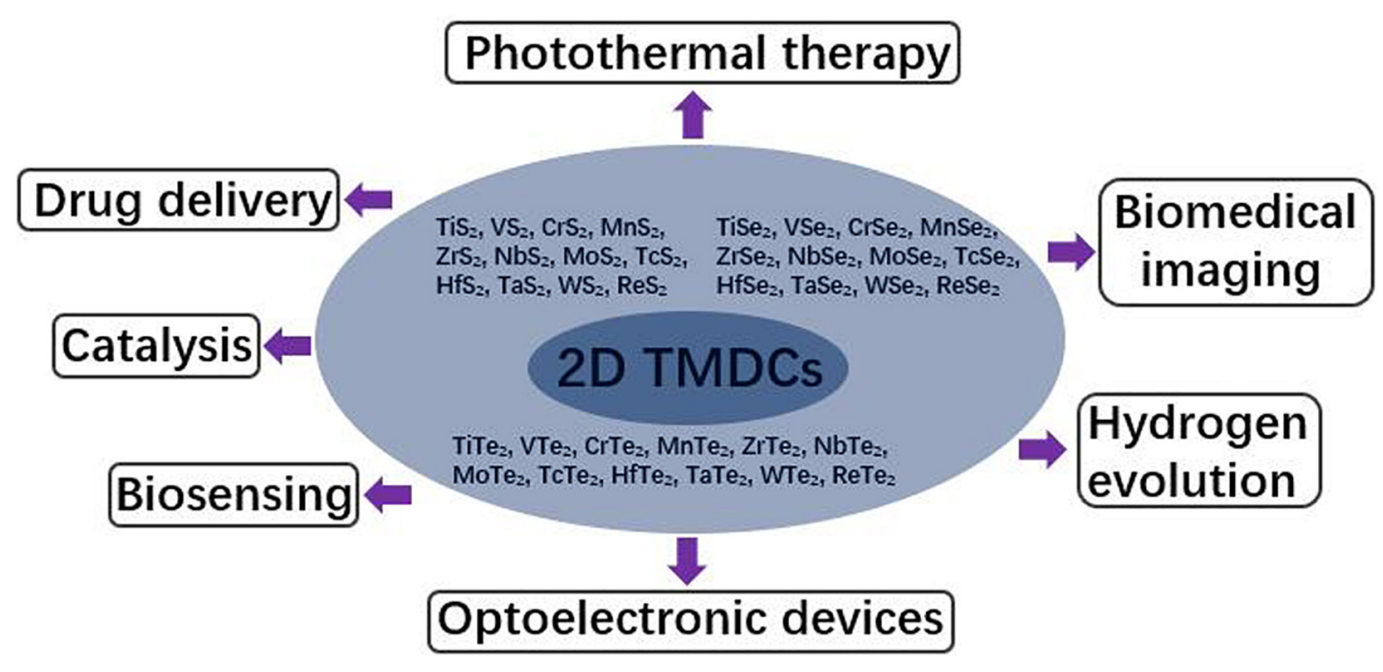

FIGURE 1 | Summary of the types and applications of 2D TMDCs.

The same as graphene and graphene derivatives, 2D TMDCs possess ultrathin structure and high surface-area-to-mass ratio (Chhowalla et al., 2013), which are favorable for loading multiple molecules, such as organic molecules (Liu et al., 2014b; Yin et al., 2014; Ariyasu et al., 2017) and genes (Kim et al., 2016), through van der Waals interaction or covalent bonds. Compared with graphene, the electronic structure of 2D TMDCs exhibit great differences. Graphene is an indirect band gap semiconductor, while the unique electronic structure of 2D TMDCs makes them direct band gap semiconductors (Chhowalla et al., 2013). In addition, only through different chemical modification, graphene can realize the diversity of properties. Two-dimensional TMDCs are a large family, including 36 kinds of materials. The band gap energy of 2D TMDCs varies with the composition of elements, and the properties of $2 \mathrm{D}$ TMDCs can be diversified without surface modification (Chhowalla et al., 2013). Thus the unique structure and photoelectric properties of 2D TMDCs make them more widely used than graphene in many fields. In the field of optoelectronics, 2D TMDCs can be used in catalysis, hydrogen evolution and a variety of optoelectronic devices, such as transistors (Zhao et al., 2019), photodetectors (Park et al., 2017), photoelectric modulators (Li et al., 2017), electrodes (Wang J. et al., 2017) and battery diaphragms (Ghazi et al., 2017). In addition, $\mathrm{MoS}_{2}$ nanosheets, the typical representative of $2 \mathrm{D}$ TMDCs, exhibits thickness-dependent photoelectric effect (Pak et al., 2018) and thickness-dependent photoacoustic signal (Chen et al., 2016). The most noteworthy is that 2D TMDCs can be excellent nanoplatform for biomedical application, such as drug delivery (Liu et al., 2014b; Han et al., 2016; Yang et al., 2018), biosensing (Zhu et al., 2013; Farimani et al., 2014; Yuan et al., 2014). High photothermal/photoacoustic conversion coefficient enables 2D TMDCs promising for photothermal therapy (Jin et al., 2003; Li et al., 2012) and biomedical imaging (Ma et al., 2017; Figure 1).

The broad application prospect of 2D TMDCs greatly increases human exposure opportunities. Two-dimensional
TMDCs as airborne particles will reach human respiratory system which are generated during production, usage, transportation and disposal of 2D TMDCs-based products. The applications of 2D TMDCs in biomedical field require their injection into circulation and reaching human organs and tissues. In addition, solar energy is the most easily available and cheapest light energy. The solar disinfection of drinking water mostly depends on the ultraviolet in solar energy, which only accounts for $4 \%$ of the solar energy, leading to the low efficiency of solar disinfection. Therefore, it is urgent to develop new materials that can harvest visible light for water disinfection, so as to accelerate the water disinfection effect of solar energy. It has been proved that fewlayered $\mathrm{MoS}_{2}$ membrane produced ROS and kill bacteria in water through absorbing 50\% solar energy (Liu et al., 2016). The application of 2D TMDCs in drinking water disinfection will increase the possibility of human contact with 2D TMDCs through digestive system. Many possible exposure pathways of 2D TMDCs will greatly increase the chance of contact with human beings. Once entering human body, 2D TMDCs will disturb the normal physiological state. The biosafety evaluation of 2D TMDCs is of great significance to human health.

The existing limited studies have shown that cell viability and some other cell behaviors, such as cell proliferation (Zou et al., 2017), oxidative stress (Yang et al., 2014), cell autophagy (Zhou et al., 2019), and metabolism (Yu Y. et al., 2017) were affected by $2 \mathrm{D}$ TMDCs. In this review, I will give a brief summary based on present progress on the synthesis and surface modification methods, biomedical applications, and biosafety evaluation of 2D TMDCs. The challenges and prospects of 2D TMDCs in synthesis and biosafety evaluation will also be discussed.

\section{SYNTHESIS OF 2D TMDCs}

At present, a variety of preparation methods including mechanical exfoliation (Novoselov et al., 2004; Li et al., 2013), 
liquid phase exfoliation (Coleman et al., 2011; Vega-Mayoral et al., 2016), chemical exfoliation (Eda et al., 2011; Zeng et al., 2011), chemical vapor deposition (Kim et al., 2012; Liu K.-K. et al., 2012; Wang et al., 2013), and solvothermal synthesis (Peng et al., 2001b; Ramakrishna Matte et al., 2010), have been developed to synthesize 2D TMDCs with single or few layers. These methods can be divided into two categories: top-down (get layered nanomaterials from bulk crystals through different exfoliation ways) and bottom-up approaches (use atoms or molecules as precursors to grow into layered nanomaterials under special conditions) (Fiori et al., 2014).

\section{Top-Down Synthesis Mechanical Cleavage}

Mechanical cleavage is the most typical top-down method. In the mechanical cleavage process, the adhesive force of scotch tape is used to obtain monolayer or few-layer structures from bulk crystals. To date, many kinds of ultrathin 2D TMDCs have been synthesized in virtue of mechanical cleavage method (Novoselov et al., 2004; Splendiani et al., 2010; Radisavljevic et al., 2011). Mechanically exfoliated ultrathin 2D TMDCs are equipped with personal advantages and disadvantages. Ultrathin 2D TMDCs prepared through this method are highly crystalline nanosheets with large size and few defects, which are suitable for electronic devices and fundamental studies of intrinsic physicochemical properties. However, the production rate is low, and the size and thickness are hard to control. The substrate is needed to support the nanosheet. The 2D TMDCs prepared by this method is difficult to meet the needs of biomedicine (Chen et al., 2015).

\section{Liquid Exfoliation}

Liquid exfoliation is another typical top-down method. Liquid exfoliation could realize successful exfoliation of bulk crystals via ultrasonication in specific solvent (Figure 2; Dines, 1975;
Joensen et al., 1986; Bang et al., 2014; Yong et al., 2014). By sonicating, the weak van der Waals interaction but not strong covalent bonds in-plane could be broken down. Therefore, proper ultrasonic intensity and ultrasonic time are critical to realize the successful exfoliation of bulk crystals. The main function of solvent molecules is to stabilize exfoliated nanosheets and inhibit their reassemble. The solvent molecules with appropriate surface energy bind to the surface of nanosheets via van der Waals interaction. Hence the matching degree of surface free energy between solvent molecules and nanosheets is very important to improve the exfoliation efficiency. At present, the common solvents are mainly organics, such as dimethylformamide (DMF) and N-methyl-pyrrolidone (NMP) (Jawaid et al., 2016). To date, multiple ultrathin 2D TMDCs have been synthesized through liquid exfoliation, such as $\mathrm{MoS}_{2}$ (Bang et al., 2014), WS $\mathrm{WS}_{2}$ (Vega-Mayoral et al., 2016), $\mathrm{NbSe}_{2}$, $\mathrm{TaSe}_{2}$, and $\mathrm{NiTe}_{2}$ (Coleman et al., 2011). Liquid exfoliation makes up for some deficiencies of mechanical cleavage, realizing the large-scale preparation of ultrathin 2D TMDCs with good photoelectric properties. However, the organic solvents used in liquid exfoliation process are undesirable in following applications, and it is difficult to produce single-layer 2D TMDCs through this method. Therefore, it is necessary to further improve the experimental conditions for the large-scale synthesis of monolayer 2D TMDCs in non-toxic solvent.

\section{Chemical Exfoliation}

Chemical exfoliation method is to insert intercalators into the interlayer of the bulk crystals with the help of ultrasonication in water, realizing the successful exfoliation of bulk crystals (Eda et al., 2011; Lukowski et al., 2013). The most common intercalators are organometallic compounds, such as butyl lithium, naphthyl sodium, etc. During the synthesis process, intercalators are firstly intercalated into interlayer of bulk
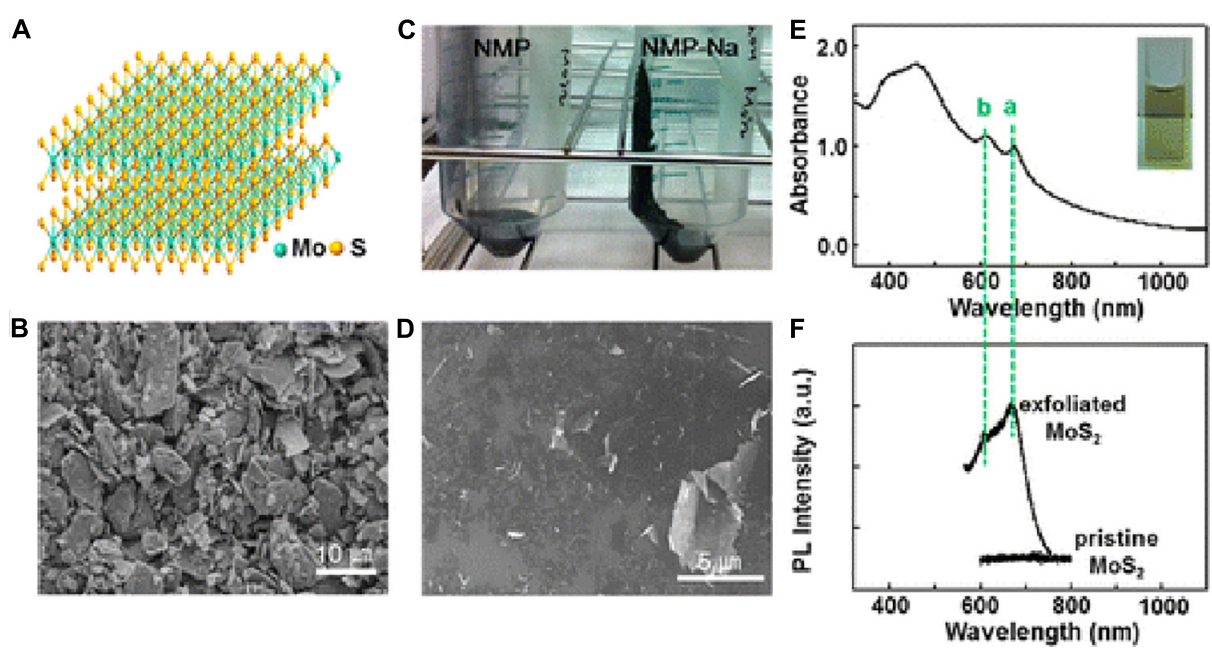

FIGURE 2 | Liquid exfoliation of bulk $\mathrm{MoS}_{2}$. (A) The structure of $\mathrm{MoS}_{2}$, (B) SEM images of pristine MoS 2 powder, (C) photo image for the effect of exfoliation with $\mathrm{NaOH}$ (the right side) and without $\mathrm{NaOH}$ (the left side, the control sample), (D) SEM image of the exfoliated MoS 2 nanosheets with NaOH in NMP, (E) UV-vis spectrum (inset: photo image of the $\mathrm{MoS}_{2}$ dispersion), and (F) photoluminescence spectrum. The "a" and "b" peaks can be assigned to the characteristics of a $2 \mathrm{H}-\mathrm{MoS}_{2}$ nanosheet and correspond to the smallest direct transition. Reproduced with permission from Bang et al. (2014). 
TMDCs in water or ethanol. Then the bulk TMDCs are exfoliated into ultrathin nanosheets under sonication. Now the insertion of intercalator into bulk TMDCs has been realized in battery, and the amount of intercalator was regulated by controlling the voltage (Zeng et al., 2011). Chemical exfoliation has been used to prepare a variety of ultrathin 2D TMDCs without the use of toxic organic solvents in the synthesis process. This method could meet the needs of the biomedical applications of ultrathin 2D TMDCs.

\section{Bottom-Up Synthesis Chemical Vapor Deposition}

Chemical vapor deposition is a typical bottom-up method. The reaction process is to expose the reaction precursor to the substrate under high temperature and pressure. The role of reaction precursors is to provide transition metal atoms and chalcogenide atoms, respectively, and react to generate ultrathin 2D TMDCs (Figure 3). Finally, the reaction product was deposited on the substrate, thus the ultrathin 2D TMDCs were obtained (Lee et al., 2012; Liu K.-K. et al., 2012; Wang et al., 2013, 2014; Ling et al., 2014). Ultrathin 2D TMDCs nanosheets prepared through this method possess excellent electronic property and high crystal quality. However, high vacuum and high temperature are necessary in the synthesis process. And the use of substrate increases the transfer process of nanosheets.

\section{Solvo-Thermal Synthesis}

Solvo-thermal synthesis is another bottom-up method. By solvothermal method, ultrathin 2D TMDCs could be obtained from precursors under the condition of specific solvent and specific reaction time (Peng et al., 2001a,b; Cao et al., 2014). The results show that after the reaction of molybdic acid or tungstic acid with thiourea at $773 \mathrm{~K}$ for $3 \mathrm{~h}$, the ultrathin $\mathrm{MoS}_{2}$ or $\mathrm{WS}_{2}$ nanosheets can be prepared. The strength of this method is that it could realize the high-yield preparation of ultrathin $2 \mathrm{D}$ TMDCs at a lower cost. Hence this method will be promising for industrial application of 2D TMDCs. The shortage of solvo-thermal synthesis is that single-layer nanosheet is difficult to be obtained.

\section{Surface Modification}

Due to the high surface area-mass-ratio, abundant atoms of 2D TMDCs are exposed to the outside, leading to super high surface free energy (Chhowalla et al., 2013). Hence the ultrathin
2D TMDCs are lack of stability in physiological conditions. To improve the stability, dispersibility and potential application in multiple fields, surface modification of 2D TMDCs are needed urgently. There are two kinds of surface modification methods (Figure 4), including physical adsorption (Figure 5; Yong et al., 2014; Han et al., 2016; Dou et al., 2017) and chemical bonding (Figure 6; Shen et al., 2016; Oudeng et al., 2018). Physical adsorption is mainly through the electrostatic attraction, hydrophobic interaction, and van der Waals force to achieve the surface modification of nanosheets. For example, doxorubicin, as a small molecule drug, could be adsorbed on the surface of $\mathrm{MoS}_{2}$ nanosheets through hydrophobic interaction and improved the efficiency of $\mathrm{MoS}_{2}$ nanosheets in killing cancer cells (Liu et al., 2014b; Yin et al., 2014). In addition to small molecules, large molecules such as DNAs and proteins can also be connected to the surface of 2D TMDCs through non-covalent interaction. The dye labeled single-strand DNA probe can be adsorbed to the surface of $\mathrm{MoS}_{2}$ nanosheets through van der Waals interaction between the bases and the surface of $\mathrm{MoS}_{2}$ nanosheets, thus realizing the effective detection of DNA (Han et al., 2016; Lu et al., 2017). After being mixed with $\mathrm{WS}_{2}$ solution for $3 \mathrm{~h}$ at room temperature, bovine serum album was successfully adsorbed on the surface of $\mathrm{WS}_{2}$ nanosheets through van der Waals interaction (Yong et al., 2014).

Chemical bonding achieves surface modification of the nanosheets with the help of covalent bond or coordination bond (Yong et al., 2014; Shen et al., 2016; Han et al., 2017; Oudeng et al., 2018). The chemical bond is much stronger than van der Waals force. At present, the commonly used chemical bonding method is to form a transition metal-sulfur bond between the molecules with sulfur atom at the end and the transition metal atom on the surface of 2D TMDCs, realizing the surface modification of 2D TMDCs. When folic acid-PEG-SH was mixed with $\mathrm{MoS}_{2}$ nanosheets for $5 \mathrm{~h}$ under ultrasonication, the surface modified $\mathrm{MoS}_{2}$ nanosheets were obtained, which could specifically target the cells over expressing folate receptor (Oudeng et al., 2018). In addition to the surface polyethylene glycol (PEG) modification of 2D TMDCs, some small molecules such as thiobarbituric acid (TBA) can also be connected to the surface of $\mathrm{MoS}_{2}$ nanosheets through the formation of Mo-S bond. Various surface modifications of $\mathrm{MoS}_{2}$ nanosheets can be realized by $\mathrm{NH}_{2}$ on TBA (Figure 6; Presolski et al., 2017). In addition, 2D TMDCs

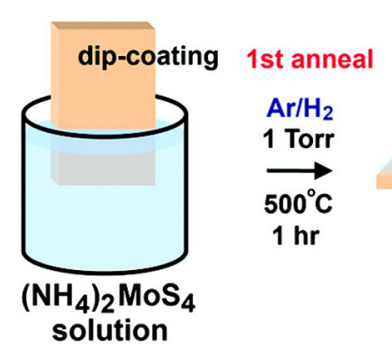

FIGURE 3 | Schematic illustration of the two-step thermolysis process for the synthesis of $\mathrm{MoS}_{2}$ thin layers on insulating substrates. The precursor $\left(\mathrm{NH}_{4}\right)_{2} \mathrm{MoS}_{4}$ was dip-coated on $\mathrm{SiO}_{2} / \mathrm{Si}$ or sapphire substrates followed by the two-step annealing process. The as-grown MoS $\mathrm{S}_{2}$ film can be transferred onto other arbitrary substrates. Reproduced with permission from Liu K.-K. et al. (2012). 


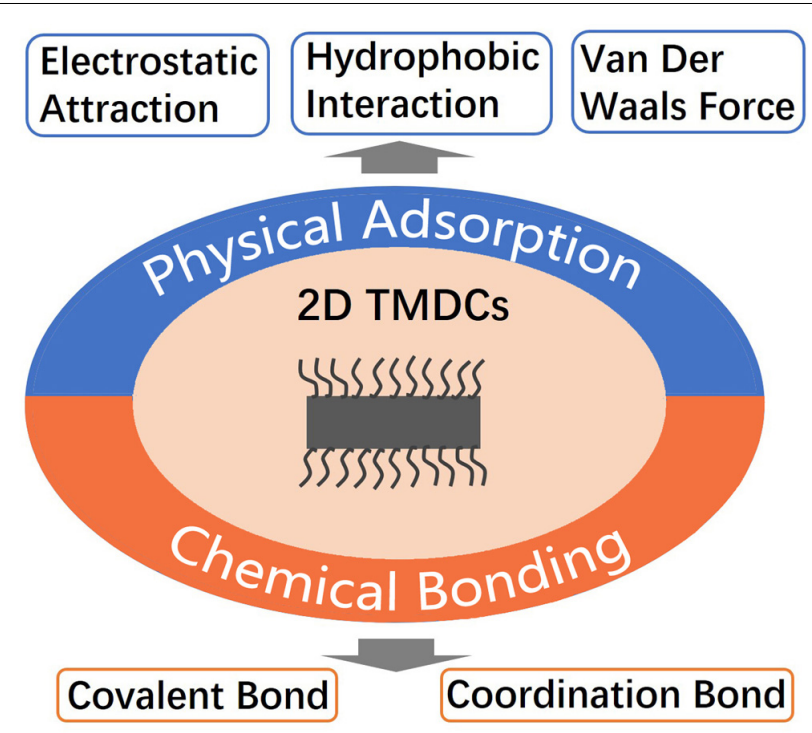

FIGURE 4 | Two kinds of surface modification methods of 2D TMDCs.

could also be functionalized by in situ polymerization with polymer (Shen et al., 2016).

The surface modification of 2D TMDCs will widen their application range and stimulate their application potential. For example, multiple functionalized 2D TMDCs have been used in drug delivery, photothermal therapy and tumor imaging. However, the functionalization methods of 2D TMDCs are insufficient. Massive efforts are still needed to complete the surface modification methods of 2D TMDCs.

\section{BIOMEDICAL APPLICATIONS OF 2D TMDCs}

In recent years, with the rapid development of preparation methods and surface functionalization methods, 2D TMDCs with various properties are on the crease, which greatly promote their application in biomedical field. Current biomedical application of 2D TMDCs can be divided into four categories: drug delivery, photothermal therapy, biological imaging, and biosensing.

\section{Drug Delivery}

As a drug carrier, 2D TMDCs has three advantages: firstly, compared with liposomes and micelles, 2D TMDCs with stronger stability can achieve sustained release of drugs and avoid explosive drug release; secondly, the super high surface area of 2D TMDC provides a large number of anchor sites for upload molecules; and thirdly, the surface decoration of $2 \mathrm{D}$ TMDCs can be achieved easily through physical adsorption or chemical bonding. Ultrathin 2D TMDCs can efficiently upload a variety of drug molecules, including doxorubicin, 7-ethyl10-hydroxycamptothecin, chitosan, photodynamic reagent, etc (Liu et al., 2014b; Yin et al., 2014; Han et al., 2016; Yang et al., 2018). For example, a functionalized $\mathrm{MoS}_{2}$ nanosheets was developed for combined cancer therapy. After $\mathrm{MoS}_{2}$ nanosheets synthesis, lipoic acid modified PEG (LA-PEG) was linked to the surface of $\mathrm{MoS}_{2}$ nanosheets to improve biocompatibility and physiological stability. The strong NIR absorbance makes the $\mathrm{MoS}_{2}$-PEG nanosheets promising candidate for photothermal therapy. Due to the super high surface-area-to-mass ratio, $\mathrm{MoS}_{2}$ nanosheets exhibited a high drug loading percent for chemotherapy drugs, such as doxorubicin, photodynamic agent chlorine e6 and 7-ethyl-10-hydroxycamptothecin. In vitro cell culture tests and in vivo cancer treatment, $\mathrm{MoS}_{2}-\mathrm{PEG}$ with doxorubicin uploaded can be utilized for chemotherapy and combined photothermal (Liu et al., 2014b). As a platform for immunotherapy, $\mathrm{MoS}_{2}-\mathrm{PEG}-\mathrm{CpG}$ was also constructed. After $\mathrm{MoS}_{2}$ nanosheets were synthesized by chemical exfoliation, the nanosheets were functionalized by cytosine-phosphateguanine $(\mathrm{CpG})$ and $\mathrm{PEG}$, and finally the $\mathrm{MoS}_{2}-\mathrm{PEG}-\mathrm{CpG}$ nanoconjugates were formed. $\mathrm{MoS}_{2}-\mathrm{PEG}-\mathrm{CpG}$ remarkably promoted the intracellular accumulation of $\mathrm{CpG}$ and stimulated the production of proinflammatory cytokines, elevating the immune response level. Not only that, when co-cultured with macrophage-like cells, $\mathrm{MoS}_{2}-\mathrm{PEG}-\mathrm{CpG}$ nanoconjugates effectively reduced the proliferation activity of cancer cells upon NIR irradiation, suggesting a new strategy for cancer treatment (Han et al., 2017). In addition to drug delivery, 2D TMDCs can also be used as an excellent gene delivery platform (Kou et al., 2014; Kim et al., 2016). Polyethylenimine (PEI) and PEG were attached to the surface of $\mathrm{MoS}_{2}$ nanosheets via disulfide bonds. Then DNA interacted with the $\mathrm{MoS}_{2}$-PEIPEG hybrid nanocomposite by electrostatic interaction, and a complex with high stability was formed. Upon near infrared light irradiation, photothermally triggered endosomal escape was induced and the polymers were detached from surface of $\mathrm{MoS}_{2}$ nanosheets by the intracellular glutathione, resulting in gene release from the hybrid nanocomposite. This sequential process significantly enhanced gene delivery efficiency without severe cytotoxicity. This $\mathrm{MoS}_{2}$ nanocomposite provided a controllable platform to deliver genes into cells (Kim et al., 2016). In another system, the amino end of $\mathrm{MoS}_{2}$-PEG-PEI nanosheets bound to negatively charged siRNA. As a critical regulator of cell cycle, a well-known oncogene Polo-like kinase 1 was investigated. After the knockdown of Polo-like kinase 1 with siRNA carried by $\mathrm{MoS}_{2}$-PEG-PEI nanosheets, the interfering efficiency and transfection effect were measured respect through qPCR, western blot and apoptosis assay. All the results suggested that as a novel nanocarrier, $\mathrm{MoS}_{2}$-PEG-PEI nanosheets exhibited high gene-carrying ability, good biocompatibility, as well as reduced cytotoxicity (Kou et al., 2014). Two-dimensional TMDCs have great potential to become excellent gene carrier. In addition, graphene, another 2D nanomaterial, which has been studied more recently, can also be used for drug loading. The drug molecule realized the drug upload through the amide reaction with the carboxyl group on the surface of graphene (Zhang M. et al., 2013, 2017). However, the smooth progress of amide reaction requires the introduction of condensation agent and dehydrating agent, which greatly increases the complexity of this process. Because 2D TMDCs have a sandwich structure, in which the metal atoms are in the middle of the layered structure 
A

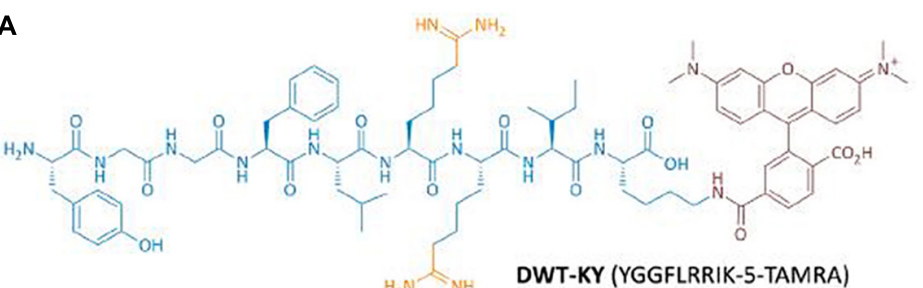

B

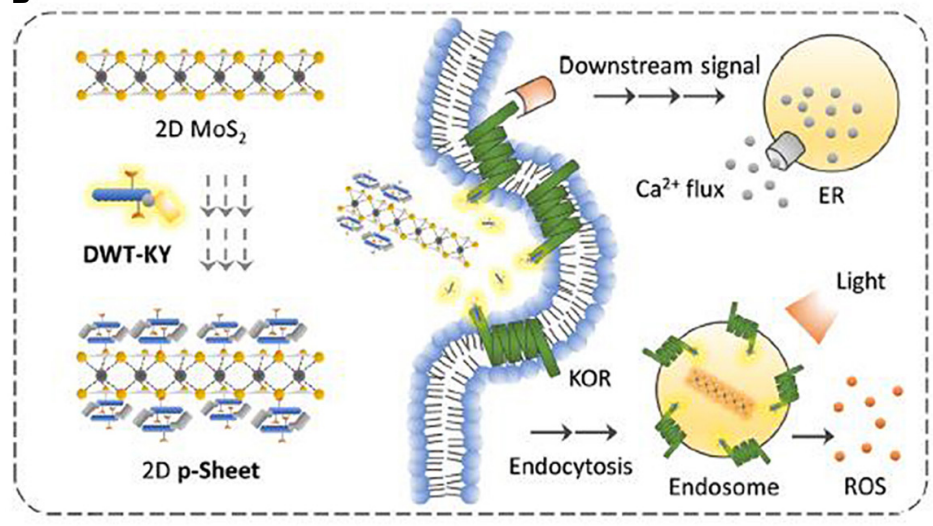

c

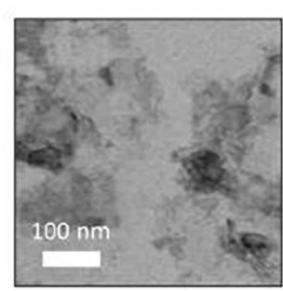

E

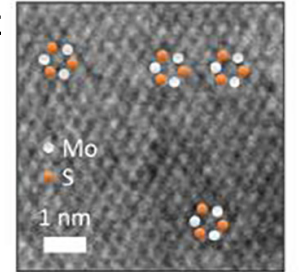

G

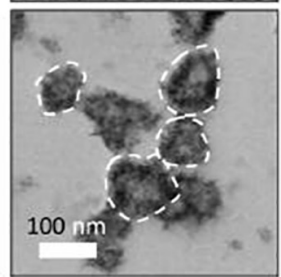

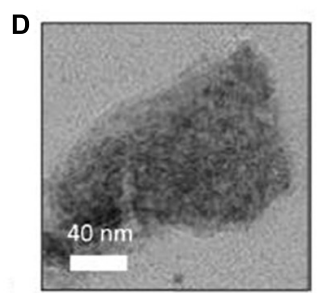

$F$

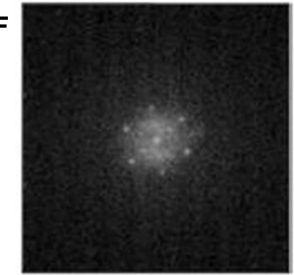

H

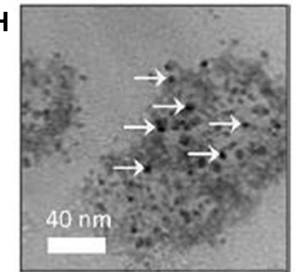

FIGURE 5 | Surface modification of 2D TMDCs through physical adsorption. (A) Structure of the fluorescent agonist probe DWT-KY (YGGFLRRIK-5-TAMRA, where TAMRA is 5-carboxu etramethylrhodamine) for KOR binding. (B) Schematic illustration of the formation of a 2D p-Sheet between 2D MoS 2 and DWT-KY and the use of the material ensemble for targeted activation of a KOR. This then leads to (1) activation of a downstream signaling pathway to release Ca ${ }^{2+}$ flux from endoplasmic reticulum and (2) endocytosis of the material that can release ROS intracellularly upon light irradiation. (C-E) High-resolution transmission electron microscopy (HRTEM) of 2D $\mathrm{MoS}_{2}$. (F) Fast Fourier transform pattern of a selected area from HRTEM of 2D MoS 2 . (G,H) HRTEM image of 2D p-Sheet (DWT-KY/2D $\mathrm{MoS}_{2}=1 \mu \mathrm{M} / 35 \mu \mathrm{g} \mathrm{mL}^{-1}$ ) [the dashed circles in $(\mathbf{G})$ highlight several representative 2D p-Sheets, and the arrows in (H) highlight several agonist probe particles adhered to the surface of 2D MoS 2 . Reproduced with permission from Dou et al. (2017).

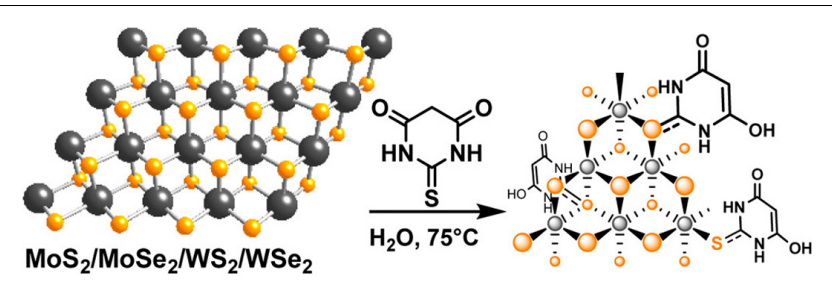

FIGURE 6 | Surface modification of 2D TMDCs through chemical bonding. Synthesis of thiobarbituric acid (TBA)-modified $\mathrm{MoS}_{2} / \mathrm{MoSe}_{2} \mathrm{WS}_{2} \mathrm{WSe}_{2}$ with high organic molecule coverage. Reproduced with permission from Presolski et al. (2017).

and the chalcogen atoms are on the surface, the drug molecules can be directly uploaded through the interaction with chalcogen atoms on the surface, and the reaction process is relatively simple (Chhowalla et al., 2013; Liu et al., 2014b). Compared with graphene, 2D TMDCs are more suitable for drug loading.

\section{Photothermal Therapy}

The principle of photothermal therapy is to use laser to generate heat and induce hyperthermia within tumor tissue, which causes denaturation of proteins, disruption of cell membrane and irreversible damage to cancer cells (Shibu et al., 2013). However, the non-specific high-intensity laser treatment damages both normal and tumor tissues, causing serious side effects (Huang et al., 2007). The introduction of photothermal agents can effectively improve the specificity of laser, which means that it can selectively generate heat within tumor tissue at a relatively low laser intensity, thus reducing the damage to normal tissue (Jin et al., 2003; Li et al., 2012). Because of strong light absorption ability in the near-infrared window area, 2D TMDCs have been used as photothermal agents in photothermal therapy and have played an effective role in removing cancer cells in vivo (Figure 7; Yin et al., 2014; Qian et al., 2015; Wang et al., 2015a; Han et al., 2017). The mass extinction coefficient of ultrathin $\mathrm{MoS}_{2}$ nanosheets with thickness of about $1.54 \mathrm{~nm}$ was $29.2 \mathrm{~L} \mathrm{~g}^{-1} \mathrm{~cm}^{-1}$, which was $7 \sim 8$ folds higher than that of graphene. When the concentration of $\mathrm{MoS}_{2}$ was between 38 and $300 \mathrm{ppm}$, the solution temperature can rapidly raised to $40^{\circ} \mathrm{C}$ by irradiation with a wave laser at $800 \mathrm{~nm}$. The in vitro studies in human cervical cancer cell line showed that almost all cells were killed after incubation with $\mathrm{MoS}_{2}$ nanosheets for $20 \mathrm{~min}$ under $800 \mathrm{~nm}$ near-infrared light (Chou et al., 2013). As another typical representative of 2D TMDCs family, $\mathrm{WS}_{2}$ nanosheets, similar to $\mathrm{MoS}_{2}$ nanosheets, also has high photothermal conversion coefficient. It was found that the mass extinction coefficient of $\mathrm{WS}_{2}$-PEG nanosheets at $808 \mathrm{~nm}$ reached $23.8 \mathrm{~L} \mathrm{~g}^{-1} \mathrm{~cm}^{-1}$, then its photothermal therapy ability was tested by in vivo experiments. After $\mathrm{WS}_{2}-\mathrm{PEG}$ nanosheets was intravenously injected into mice, when irradiated with $808 \mathrm{~nm}$ laser at the intensity of $0.8 \mathrm{~W} \mathrm{~cm}^{-2}$, the tumor 


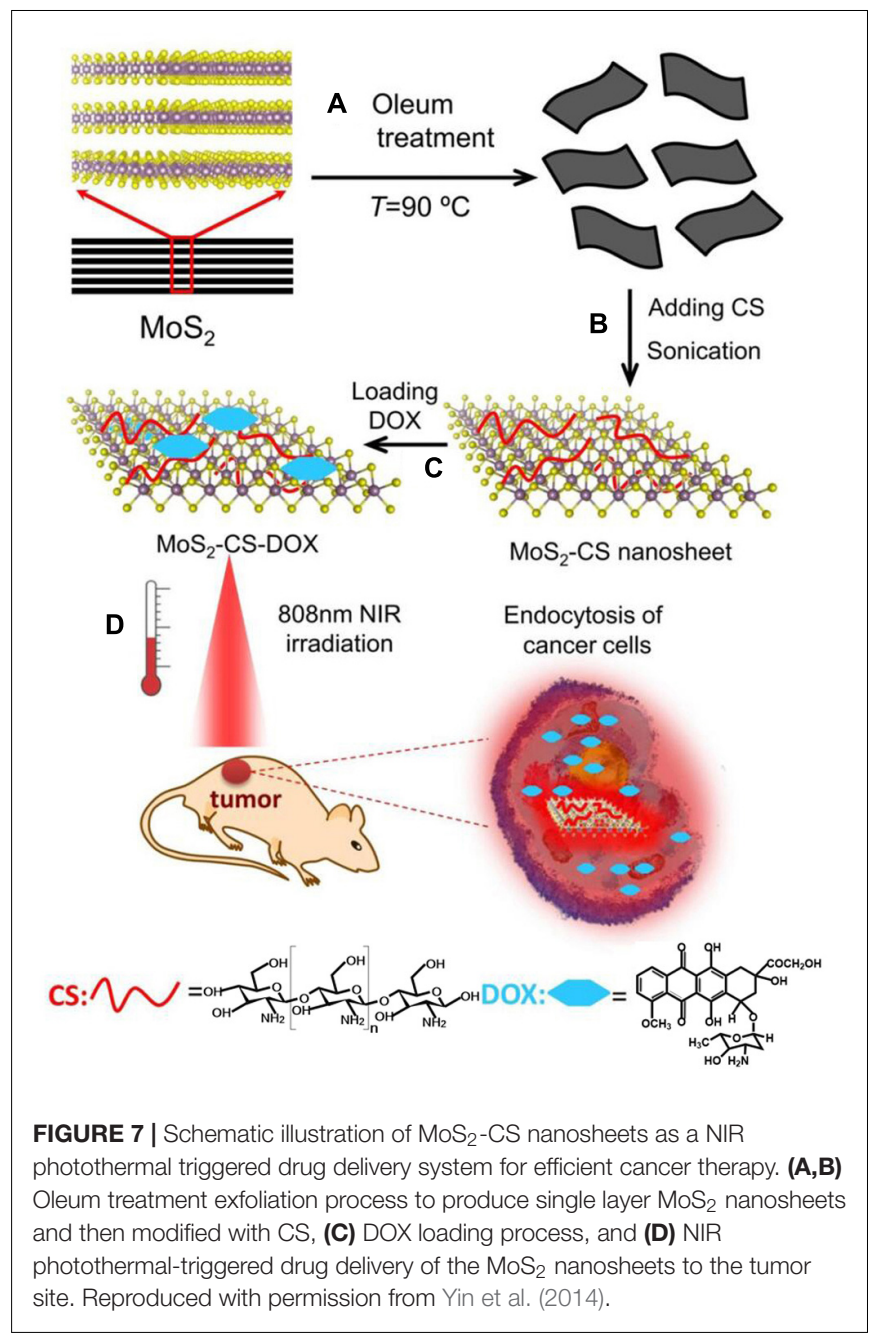

surface temperature of mice reached $65^{\circ} \mathrm{C}$ within $5 \mathrm{~min}$, and the tumor could be completely removed without obvious recurrence, thus greatly improving the survival rate of mice (Cheng et al., 2014). In addition to $2 \mathrm{D}$ TMDCs, $2 \mathrm{D}$ graphene and graphene derivatives can also be used in photothermal therapy because of their high light absorption efficiency in the near-infrared window (Liu W. et al., 2019; Roy et al., 2019). Compared with graphene, the mass extinction coefficient of $\mathrm{MoS}_{2}$ nanosheets with thickness of $1.54 \mathrm{~nm}$ was 7.8 times that of graphene in the near infrared range, which suggested that 2D TMDCs may be a better choice than graphene in photothermal treatment (Chou et al., 2013).

\section{Biomedical Imaging}

Based on the unique chemical composition and the special physical and chemical properties of layered structure, 2D TMDCs can be effectively used in a variety of biological imaging. At present, the application of 2D TMDCs in the field of biological imaging can be divided into three categories: fluorescence labeled imaging, photoacoustic imaging and X-ray computed tomography (CT) imaging. When $2 \mathrm{D}$ TMDCs are used for fluorescence labeled imaging, it is necessary to label 2D TMDCs with fluorescent molecules, and then the fluorescent imaging of cells or tissues can be realized by targeting specific cells or tissues with fluorescent labeled nanosheets (Dou et al., 2017; Ma et al., 2017). For example, after the peptide ligand (TAMRA $\mathrm{DN} 1 \mathrm{~K}$ ) linked with the fluorophore group was connected to $\mathrm{MoS}_{2}$ nanosheets, the nanosheets can recognize the liver cancer cells or liver cancer tissues with high expression of CD47. At this time, TAMRA DN1K on $\mathrm{MoS}_{2}$ nanosheets combined with CD47 on cell surface, realizing the fluorescence imaging of the cancer cells or tissues with high expression of CD47 (Ma et al., 2017). Photoacoustic imaging tomography (PAT) is a new biomedical imaging modality. Materials with light absorption capacity can produce sound waves. The principle of photoacoustic imaging is based on the photoacoustic effect of light-absorbers. Compared with the traditional in vivo optical imaging, photoacoustic imaging significantly enhanced imaging depths and spatial resolution (Wang and $\mathrm{Hu}, 2012$ ). Due to the strong absorbance in the near-infrared region, 2D TMDCs could realize effective photoacoustic signal conversion, and be used as a light absorbent for photoacoustic imaging. For example, after being intravenously injected with $\mathrm{WS}_{2}-\mathrm{PEG}$ nanosheets, the mice bearing 4T1-tumors were imaged under a PAT imaging system with a $700 \mathrm{~nm}$ laser as excitation source. Remarkably enhanced PA signals were observed in the area of tumor, which indicating more tumor accumulation of $\mathrm{WS}_{2}$-PEG nanosheets (Cheng et al., 2014). In addition, $\mathrm{TiS}_{2}$ nanosheets with appropriate surface modification could also be effectively used in vivo photoacoustic imaging, and enhanced the efficiency of subsequent photothermal therapy (Qian et al., 2015). Since the mass extinction coefficient of graphene is far less than that of $2 \mathrm{D}$ TMDCs, and only materials with strong light absorption ability can generate sound waves, compared with 2D TMDCs, graphene is not a better choice for photoacoustic imaging (Chou et al., 2013). As a widely used imaging technology, X-ray CT imaging has the advantages of high resolution and deep tissue penetration. The principle of X-ray CT imaging is to reduce the intensity of $\mathrm{X}$-ray and improve the contrast imaging ability by the strong X-ray attenuation ability of contrast agent (Shilo et al., 2012). Multiple nanomaterials containing elements with high atomic number have good X-ray attenuation ability (Liu et al., 2012a; Liu Z. et al., 2012). Due to the high atomic number and the strong X-ray attenuation ability of transition metal, 2D TMDCs are excellent contrast agents for X-ray CT imaging. After intravenous injection, both chitosan modified $\mathrm{MoS}_{2}$ (Yin et al., 2014) and $\mathrm{WS}_{2}$-PEG nanosheets (Cheng et al., 2014) exhibited excellent X-ray CT imaging ability in mice. Compared with 2D TMDCs, the X-ray attenuation ability of carbon element with smaller atomic number is weaker, so graphene is difficult to be a better contrast agent for X-ray CT imaging. In previous studies, graphene must be combined with other nanomaterials to form a composite material, which could be used in X-ray CT imaging (Zhang Y. et al., 2017). For example, after depositing graphene oxide on the surface of microcapsule containing gold nanoparticles, this microcapsule could serve as effective contrast agent to enhance $\mathrm{X}$-ray $\mathrm{CT}$ imaging in vitro and in vivo (Jin et al., 2013). 


\section{Biosensing}

As a novel biosensing platform, 2D TMDCs have two significant advantages. On the one hand, the super high surface area of $2 \mathrm{D}$ planar structure can fix a large number of sensing molecules to reach a very low detection limit. On the other hand, 2D TMDCs can excite the fluorescence group to the conduction band of $2 \mathrm{D}$ nanosheets through the photoinduced electron transfer effect, realizing the fluorescence quenching effect. In sum, 2D TMDCs are expected to be more advantageous biosensing platforms for detection of DNA (Figure 8; Zhu et al., 2013; Farimani et al., 2014; Yuan et al., 2014; Dou et al., 2017; Lu et al., 2017) and other small molecules (Xi et al., 2014). The fluorescent moleculeslabeled single-strand DNA can be adsorbed on the surface of the nanosheets through the van der Waals interaction between the nucleobases and the basal plane of $\mathrm{MoS}_{2}$ nanosheets, which makes the single-strand DNA fluorescence quenching. When single-strand DNA was hybridized with its complementary DNA to form double-strand DNA, the interaction between the singlestrand DNA and $\mathrm{MoS}_{2}$ nanosheets were greatly weakened. The single-strand DNA separated from the $\mathrm{MoS}_{2}$ nanosheets and the fluorescence quenched by $\mathrm{MoS}_{2}$ nanosheets can be retained, successfully detecting DNA molecules by $\mathrm{MoS}_{2}$ nanosheets. The detection limit of the biosensor based on $\mathrm{MoS}_{2}$ nanosheets for DNA was $500 \mathrm{PM}$ and $5 \mu \mathrm{m}$ for adenosine. This homogeneous process can be completed within a few minutes, showing a great application prospect of 2D TMDCs in biosensing and molecular diagnosis (Zhu et al., 2013).

\section{BIOSAFETY EVALUATION OF 2D TMDCs}

Two-dimensional TMDCs have emerged as promising materials for catalysis (Lukowski et al., 2013), photoelectronic devices (Yu P. et al., 2017; Si et al., 2018), energy storage (Wang T. et al., 2017), biosensing (Zhu et al., 2013), photodynamic therapy (Liu et al., 2014a; Yong et al., 2014), drug and gene delivery (Liu et al., 2014b; Kim et al., 2016; Ariyasu et al., 2017; Ma et al., 2017), due to various unique optical, electronic, mechanical,

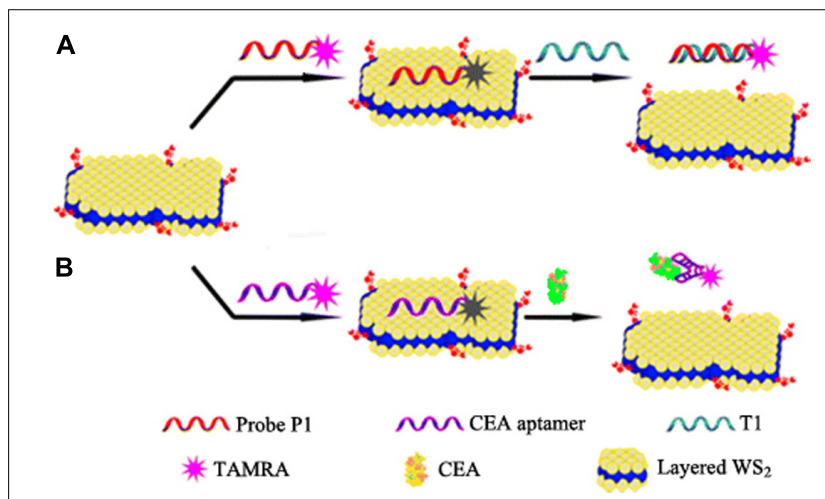

FIGURE 8 | Schematic illustration of fluorescence sensing of nucleic acid and protein with layered $\mathrm{WS}_{2}$ nanosheet as the quencher. Two biosensors utilizing two different target recognition models, i.e., a nucleic acid hybridization model (A) and a protein-aptamer reaction model (B), respectively. Reproduced with permission from Yuan et al. (2014). and chemical properties. The increasing applications of $2 \mathrm{D}$ TMDCs have increased their environmental accumulation and the possibilities of human exposures. Two-dimensional TMDCs as airborne particles, which are generated during production, usage, transportation and disposal of 2D TMDC-based products, will reach human respiratory system. In addition, the applications of 2D TMDCs in biomedical field enable their direct injection into circulation and accumulation in human organs and tissues. As foreign materials, 2D TMDCs certainly will disturb the normal physiological state. The biosafety evaluation of $2 \mathrm{D}$ TMDCs is of great significance to human health. The investigations on the biosafety of 2D TMDCs is still in its infancy. In this part, we make a summary on the basis of present research.

Cell death is the most serious consequence caused by nanoparticles. Compared to other disturbances of cell function caused by $2 \mathrm{D}$ TMDCs, research on cell viability affected by 2D TMDCs started earlier. For 2D TMDCs without surface modification, cell viability measurement showed a big difference between $\mathrm{MoS}_{2}, \mathrm{WS}_{2}$, and $\mathrm{WSe}_{2}$ nanosheets. The degree of cytotoxicity can be ranked in the order of $\mathrm{WS}_{2}<\mathrm{MoS}_{2}<\mathrm{WSe}_{2}$ (Teo et al., 2014). A possible cause of this situation is that the chalcogens are mainly located on exterior of each $2 \mathrm{D}$ TMDCs layer, allowing more interaction with cells compared with transition metal. Likewise to the case between $\mathrm{H}_{2} \mathrm{Se}$ and $\mathrm{H}_{2} \mathrm{~S}$, $\mathrm{H}_{2} \mathrm{Se}$ is much more toxic than $\mathrm{H}_{2} \mathrm{~S}$ (Shamberger, 1981), selenium might be more hazardous than sulfur in 2D TMDCs, thus explaining above results. Compared with the $2 \mathrm{D}$ graphene, the cytotoxicity of various 2D TMDCs is lower than that of graphene (Teo et al., 2014). In theoretical simulation, graphene cut and inserted into cell membranes and extracted phospholipids, causing physical damage (Shi et al., 2016). However, when $\mathrm{MoS}_{2}$ nanoparticles interacted with cells, they sank into the phospholipid bilayer of cell membrane in a way parallel to the cell membrane, hardly causing physical damage to the cell membrane (Zhou et al., 2019). We speculate that the sharp edge of graphene may be the main reason for its high cytotoxicity. However, more deeply research is needed to determine if this speculation is valid. Under the same thickness and size, chemical composition was probably the major factor contributing to the variation in cytotoxicity of 2D TMDCs.

In addition to chemical composition, surface modification also produced an effect on the in vitro toxicity of $2 \mathrm{D}$ TMDCs. After incubation with $\mathrm{MoS}_{2}$ or chitosan-functionalized $\mathrm{MoS}_{2}$, the viabilities of two kinds of human cells showed that chitosanfunctionalized $\mathrm{MoS}_{2}$ was more biocompatible than unmodified $\mathrm{MoS}_{2}$ nanosheets, indicating the significance of chitosan functionalization in decreasing cytotoxicity of 2D TMDCs (Yin et al., 2014). For $\mathrm{WS}_{2}$ nanosheets, another typical 2D TMDCs, surface modification also greatly reduced the toxicity in vitro. After incubation with three kinds of human cells, respectively, PEGylated $\mathrm{WS}_{2}$ exhibited negligible cytotoxicity. In contrast, without PEGylated $\mathrm{WS}_{2}$ nanosheets were obviously toxic to human cells. After treated with unmodified $\mathrm{WS}_{2}$ nanosheets, the cell survival rates of three kinds of human cells were less than $50 \%$. This clearly suggested that the cytotoxicity of $\mathrm{WS}_{2}$ nanosheets was also closely associated with surface modification, which was consistent with $\mathrm{MoS}_{2}$ nanosheets (Cheng et al., 2014). 
In addition, several other surface-modified 2D TMDCs exhibited very low cytotoxicity even at higher concentrations in different human cells (Yong et al., 2014; Wang et al., 2015b; Yu et al., 2015; Ariyasu et al., 2017). Based on the existing limited research, it is probably that the surface modification could significantly decreased the cytotoxicity of 2D TMDCs. The specific reasons of this phenomenon need further study.

As an important physicochemical property of $2 \mathrm{D}$ nanomaterials, thickness played critical role in cell death induced by 2D TMDCs. After obtaining three $\mathrm{MoS}_{2}$ nanosheets of different thicknesses by different exfoliation methods, the in vitro toxicity of these three nanosheets to human lung cells was compared. Tert-butyllithium and n-butyllithium exfoliated $\mathrm{MoS}_{2}$ nanosheets were more cytotoxic than methyllithium exfoliated $\mathrm{MoS}_{2}$. Tert-butyllithium and n-butyllithium provided more efficient exfoliation than methyllithium. In other words, thickness was a factor influencing the cytotoxicity of $\mathrm{MoS}_{2}$ nanosheets. The smaller the thickness of the $\mathrm{MoS}_{2}$ nanosheets, the stronger their cytotoxic influence (Chng et al., 2014). The increase in active edge sites and surface area might contribute to the increased cytotoxicity caused by thickness reduction.

However, the interaction between 2D TMDCs and cells certainly will disturb cell homeostasis. Further biosafety evaluation indicated that 2D TMDCs affected cell behaviors, such as cell proliferation, differentiation (Zou et al., 2017), cell adhesion, spreading (Suhito et al., 2017), oxidative stress (Yang et al., 2014), cell metabolism (Yu Y. et al., 2017), and cell autophagy (Zhou and Yan, 2019; Zhou et al., 2019). Singlelayer $\mathrm{MoS}_{2}$ nanosheets promoted proliferation and accelerated myogenic differentiation in human embryonic lung fibroblasts (HELFs). The immunoblot assay and immunofluorescence analysis indicated the Akt-mTOR-p70S6K signaling pathway played a critical role in the induction of cellular proliferation and differentiation by single-layer $\mathrm{MoS}_{2}$ in HELFs (Zou et al., 2017). Based on the observation of cell morphology, human mesenchymal stem cells on $\mathrm{MoS}_{2}$ film exhibited more active filopodial interaction with substrate compared to the control group, indicating that $\mathrm{MoS}_{2}$ highly enhanced cell adhesion and spreading (Suhito et al., 2017). In human hepatoma HepG2 cells, $\mathrm{MoS}_{2}$ nanosheets induced mitochondrial depolarization and elevated reactive oxygen species level (Liu et al., 2017). High levels of oxidative stress induced by single-layer $\mathrm{MoS}_{2}$ nanosheets caused disturbance to Akt related signaling pathway (Zou et al., 2017) and enhanced antibacterial activity (Yang et al., 2014). In addition, chitosan functionalized $\mathrm{MoS}_{2}$ nanosheets activated TGF- $\beta /$ Smad pathway and disturbed cellular metabolic process through interacting with EGFR on human dermal fibroblasts cells surface (Yu Y. et al., 2017). $\mathrm{WS}_{2}$ nanosheets induced obvious alterations in metabolic pathways and metabolites and the phase composition made a difference on metabolomics in algae (Yuan et al., 2018). Both the cell surface adhesion of thinner $\mathrm{MoS}_{2}$ nanosheets and cell internalization of thicker $\mathrm{MoS}_{2}$ nanosheets activated mTOR-dependent autophagy signaling pathway through perturbing cell surface protein amyloid precursor proteins (Figure 9; Zhou et al., 2019).

This phenomenon of 2D TMDCs is similar to that of graphene, which can also cause different degrees of cell function disturbance in a variety of cells, including ROS, proliferation and apoptosis (Pelin et al., 2018; Alsaedi et al., 2019; Liu X. et al., 2019). For example, graphene induced autophagy by activating NFKB and TLR signaling pathways in THP-1 cells and RAW 264.7 cells, respectively. Although both graphene and 2D TMDCs induced cell autophagy, the specific upstream initiation approaches were different (Chen et al., 2012; Qin et al., 2015). We speculate that the size, thickness, surface modification, and element composition may contribute to the differences between graphene and 2D TMDC in the disturbance of cell function. Preliminary evaluation has demonstrated that in the case of low cytotoxicity 2D TMDCs caused disturbances to multiple basic cell behaviors. However, the existing research disregarded the effects
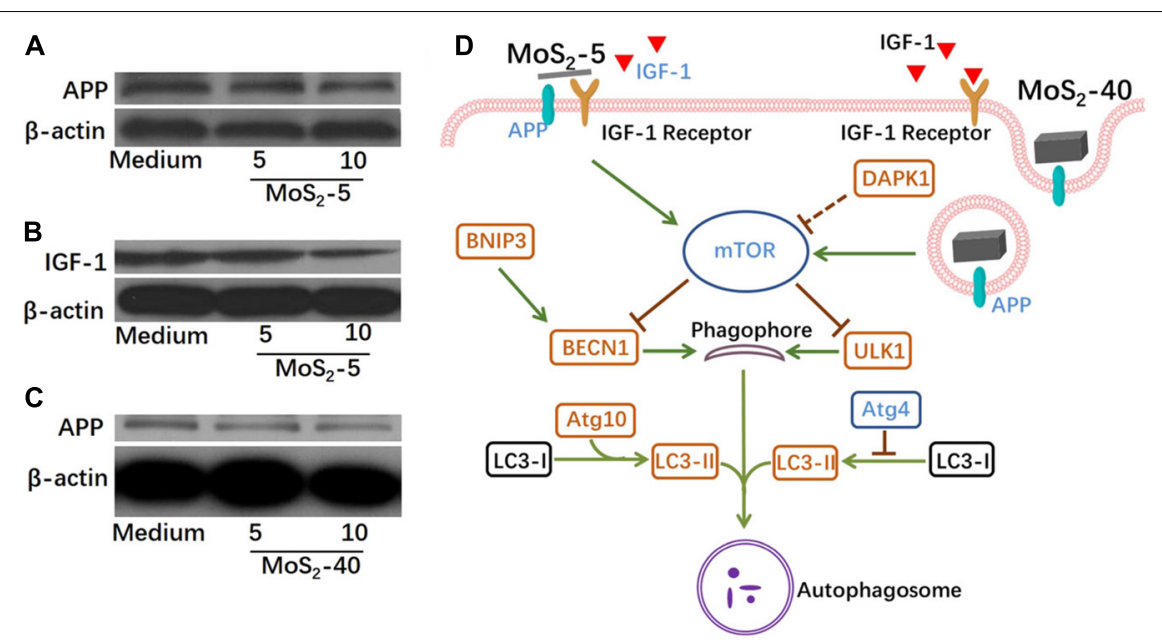

FIGURE 9 | Probable interactions between $\mathrm{MoS}_{2}$ nanosheets and cell surface proteins and the possible perturbations of autophagy-related cell signaling. Immunoblot assay indicated that $\mathrm{MoS}_{2}-5$ inhibited APP (A), IGF-1 (B), and MoS -40 inhibited APP (C). (D) Signaling scheme showing the current understanding of the probable mechanisms for $\mathrm{MoS}_{2}$-5- or $\mathrm{MoS}_{2}-40$-induced autophagy. Reproduced with permission from Zhou et al. (2019). 
of cell line types and physicochemical properties of nanosheets on the cell perturbation induced by 2D TMDCs. More systematic biological and biosafety evaluation of 2D TMDCs are urgently required to further determine their potential risks and ensure their safety application.

\section{CONCLUSION AND PESPECTIVE}

This review summarized the synthesis methods, modification methods, important biomedical applications, and biosafety evaluation of 2D TMDCs. Driven by the wide application prospect of 2D TMDCs, remarkable progress has been made in their synthesis methods in recent years. At the same time, in order to obtain 2D TMDCs with various properties and realize their application in multiple fields, more and more attention has been paid on the surface modification of $2 \mathrm{D}$ TMDCs. The maturity of synthesis and modification methods promoted the wide application of 2D TMDCs in many fields, especially in biomedical field, increasing the opportunities of human exposure. As we all know, once entering human body, 2D TMDCs may interact with biological system and disturb homeostasis of physiological system. 2D TMDCs, as a kind of nanomaterial, can also pose a threat to human health. Therefore, it is necessary to evaluate the biosafety of 2D TMDCs. As 2D graphene analogs, most of studies on 2D TMDCs have been done just in the past few years, so the research in this field is still in its infancy. To further facilitate advances of this field, there are still several critical issues to be solved.

From the perspective of nanomaterials, the synthesis of $2 \mathrm{D}$ TMDCs lacks the standard method of controllability. It is still a big challenge to synthesize 2D TMDCs with desirable size and thickness. The size and thickness of 2D TMDCs obtained by the existing synthesis method are in a distribution range. Therefore, it is necessary to develop new synthetic methodologies of $2 \mathrm{D}$ TMDCs with desirable structural and compositional parameters. In addition, the investigations on the surface modification of $2 \mathrm{D}$ TMDCs have just been carried out. In order to realize the surface diversity modification of 2D TMDCs, it is essential to clarify other surface modification methods to expand the application space of 2D TMDCs.

From the biosafety evaluation point of view, a large number of studies have shown that the disturbance of the physiological

\section{REFERENCES}

Alsaedi, I. I. J., Taqi, Z. J., Abdul Hussien, A. M., Sulaiman, G. M., and Jabir, M. S. (2019). Graphene nanoparticles induces apoptosis in MCF-7 cells through mitochondrial damage and NF-KB pathway. Mater. Res. Express 6:095413. doi: 10.1088/2053-1591/ab33af

Ariyasu, S., Mu, J., Zhang, X., Huang, Y., Yeow, E. K. L., Zhang, H., et al. (2017). Investigation of thermally induced cellular ablation and heat response triggered by planar MoS2-based nanocomposite. Bioconj. Chem. 28, 1059-1067. doi: 10.1021/acs.bioconjchem.6b00741

Bang, G. S., Nam, K. W., Kim, J. Y., Shin, J., Choi, J. W., and Choi, S.-Y. (2014). Effective liquid-phase exfoliation and sodium ion battery application of MoS2 nanosheets. ACS Appl. Mater. Inter. 6, 7084-7089. doi: 10.1021/am 4060222 system caused by nanomaterials is closely related to the properties of nanomaterials. As a new kind of 2D nanomaterials, 2D TMDCs is very different from other nanomaterials. The research on the disturbance of $2 \mathrm{D}$ TMDCs to physiological system is still in its infancy. It is still unknown how the physicochemical properties, such as element composition, size, surface charge, and hydrophobicity, will affect the biological systems disturbance caused by 2 D TMDCs. Hence it is necessary to systematically study how the various physiochemical properties affect the interaction between 2D TMDCs and physiological system and the specific molecular mechanism. On the other hand, the ultimate goal of the biosafety evaluation of 2D TMDCs is to reveal the potential risks of $2 \mathrm{D}$ TMDCs to human health. The conclusions obtained in vitro need to be further verified by in vivo experiments. Due to the complexity of the internal environment, the dosage, administration time, administration mode and model animals should be fully considered in the specific study. Finally, the possible physiological disturbance caused by $2 \mathrm{D}$ TMDCs and the specific effects of physicochemical properties are clarified through the systematic study on the internal level.

\section{AUTHOR CONTRIBUTIONS}

$\mathrm{XZ}$ designed this work of review, performed the literature search of the databases, and wrote the manuscript. HS and XB revised the manuscript. All authors approved the manuscript for publication.

\section{FUNDING}

This work was supported by the Special Scientific Research Fund for Talents Introduced of Hebei Agricultural University (YJ2019030) and the Key R\&D Programmes of Zibo (2019ZC010106).

\section{ACKNOWLEDGMENTS}

We thank Gaoxing Su and Jianbo Jia for technical assistance.

Cao, S., Liu, T., Hussain, S., Zeng, W., Peng, X., and Pan, F. (2014). Hydrothermal synthesis of variety low dimensional WS2 nanostructures. Mater. Lett. 129, 205-208. doi: 10.1016/j.matlet.2014.05.013

Chen, G.-Y., Yang, H.-J., Lu, C.-H., Chao, Y.-C., Hwang, S.-M., Chen, C.-L., et al. (2012). Simultaneous induction of autophagy and toll-like receptor signaling pathways by graphene oxide. Biomaterials 33, 6559-6569. doi: 10 . 1016/j.biomaterials.2012.05.064

Chen, J., Liu, C., Hu, D., Wang, F., Wu, H., Gong, X., et al. (2016). SingleLayer MoS2 nanosheets with amplified photoacoustic effect for highly sensitive photoacoustic imaging of orthotopic brain tumors. Adv. Funct. Mater. 26, 8715-8725. doi: 10.1002/adfm.201603758

Chen, Y., Tan, C., Zhang, H., and Wang, L. (2015). Two-dimensional graphene analogues for biomedical applications. Chem. Soc. Rev. 44, 2681-2701. doi: 10.1039/C4CS00300D 
Cheng, L., Liu, J., Gu, X., Gong, H., Shi, X., Liu, T., et al. (2014). PEGylated WS2 nanosheets as a multifunctional theranostic agent for in vivo dual-modal CT/photoacoustic imaging guided photothermal therapy. Adv. Mater. 26, 1886-1893. doi: 10.1002/adma.201304497

Chhowalla, M., Shin, H. S., Eda, G., Li, L.-J., Loh, K. P., and Zhang, H. (2013). The chemistry of two-dimensional layered transition metal dichalcogenide nanosheets. Nat. Chem. 5, 263-275. doi: 10.1038/nchem.1589

Chng, E. L. K., Sofer, Z., and Pumera, M. (2014). MoS2 exhibits stronger toxicity with increased exfoliation. Nanoscale 6, 14412-14418. doi: 10.1039/ C4NR04907A

Chou, S. S., Kaehr, B., Kim, J., Foley, B. M., De, M., Hopkins, P. E., et al. (2013). Chemically exfoliated MoS2 as near-infrared photothermal agents. Angew. Chem. Int. Ed. 52, 4160-4164. doi: 10.1002/anie.201209229

Coleman, J. N., Lotya, M., O'neill, A., Bergin, S. D., King, P. J., Khan, U., et al. (2011). Two-dimensional nanosheets produced by liquid exfoliation of layered materials. Science 331, 568-571. doi: 10.1126/science.119 4975

Dines, M. B. (1975). Lithium intercalation via n-Butyllithium of the layered transition metal dichalcogenides. Mater. Res. Bull. 10, 287-291. doi: 10.1016/ 0025-5408(75)90115-4

Dou, W.-T., Kong, Y., He, X.-P., Chen, G.-R., Zang, Y., Li, J., et al. (2017). GPCR activation and endocytosis induced by a 2D material agonist. ACS Appl. Mater. Inter. 9, 14709-14715. doi: 10.1021/acsami.7b02754

Eda, G., Yamaguchi, H., Voiry, D., Fujita, T., Chen, M., and Chhowalla, M. (2011). Photoluminescence from chemically exfoliated MoS2. Nano Lett. 11, 5111-5116. doi: 10.1021/nl201874w

Farimani, A. B., Min, K., and Aluru, N. R. (2014). DNA base detection using a single-layer MoS2. ACS Nano 8, 7914-7922. doi: 10.1021/nn5029295

Fiori, G., Bonaccorso, F., Iannaccone, G., Palacios, T., Neumaier, D., Seabaugh, A., et al. (2014). Electronics based on two-dimensional materials. Nat. Nanotechnol. 9, 768-779. doi: 10.1038/nnano.2014.207

Ghazi, Z. A., He, X., Khattak, A. M., Khan, N. A., Liang, B., Iqbal, A., et al. (2017). MoS2/Celgard separator as efficient polysulfide barrier for long-life lithiumsulfur batteries. Adv. Mater. 29:1606817. doi: 10.1002/adma.201606817

Han, J., Xia, H., Wu, Y., Kong, S. N., Deivasigamani, A., Xu, R., et al. (2016). Singlelayer MoS2 nanosheet grafted upconversion nanoparticles for near-infrared fluorescence imaging-guided deep tissue cancer phototherapy. Nanoscale 8, 7861-7865. doi: 10.1039/C6NR00150E

Han, Q., Wang, X., Jia, X., Cai, S., Liang, W., Qin, Y., et al. (2017). CpG loaded MoS2 nanosheets as multifunctional agents for photothermal enhanced cancer immunotherapy. Nanoscale 9, 5927-5934. doi: 10.1039/C7NR01460K

Huang, X., Jain, P. K., El-Sayed, I. H., and El-Sayed, M. A. (2007). Plasmonic photothermal therapy (PPTT) using gold nanoparticles. Lasers Med. Sci. 23:217. doi: 10.1007/s10103-007-0470-x

Jawaid, A., Nepal, D., Park, K., Jespersen, M., Qualley, A., Mirau, P., et al. (2016). Mechanism for liquid phase exfoliation of MoS2. Chem. Mater. 28, 337-348. doi: 10.1021/acs.chemmater.5b04224

Jin, R., Charles Cao, Y., Hao, E., Métraux, G. S., Schatz, G. C., and Mirkin, C. A. (2003). Controlling anisotropic nanoparticle growth through plasmon excitation. Nature 425, 487-490. doi: 10.1038/nature02020

Jin, Y., Wang, J., Ke, H., Wang, S., and Dai, Z. (2013). Graphene oxide modified PLA microcapsules containing gold nanoparticles for ultrasonic/CT bimodal imaging guided photothermal tumor therapy. Biomaterials 34, 4794-4802. doi: 10.1016/j.biomaterials.2013.03.027

Joensen, P., Frindt, R. F., and Morrison, S. R. (1986). Single-layer MoS2. Mater. Res. Bull. 21, 457-461. doi: 10.1016/0025-5408(86)90011-5

Kim, J., Kim, H., and Kim, W. J. (2016). Single-layered MoS2-PEI-PEG nanocomposite-mediated gene delivery controlled by photo and redox stimuli. Small 12, 1184-1192. doi: 10.1002/smll.201501655

Kim, K. K., Hsu, A., Jia, X., Kim, S. M., Shi, Y., Hofmann, M., et al. (2012). Synthesis of monolayer hexagonal boron nitride on $\mathrm{Cu}$ foil using chemical vapor deposition. Nano Lett. 12, 161-166. doi: 10.1021/nl203249a

Kou, Z., Wang, X., Yuan, R., Chen, H., Zhi, Q., Gao, L., et al. (2014). A promising gene delivery system developed from PEGylated MoS2 nanosheets for gene therapy. Nanoscale Res. Lett. 9:587. doi: 10.1186/1556-276X-9-587

Lee, Y.-H., Zhang, X.-Q., Zhang, W., Chang, M.-T., Lin, C.-T., Chang, K.-D., et al. (2012). Synthesis of large-area MoS2 atomic layers with chemical vapor deposition. Adv. Mater. 24, 2320-2325. doi: 10.1002/adma.201104798
Li, B. W., Zu, S., Zhou, J. D., Jiang, Q., Du, B. W., Shan, H. Y., et al. (2017). Singlenanoparticle plasmonic electro-optic modulator based on MoS2 monolayers. ACS Nano 11, 9720-9727. doi: 10.1021/acsnano.7b05479

Li, H., Lu, G., Wang, Y., Yin, Z., Cong, C., He, Q., et al. (2013). Mechanical exfoliation and characterization of single- and few-layer nanosheets of WSe2, TaS2, and TaSe2. Small 9, 1974-1981. doi: 10.1002/smll.201202919

Li, M., Yang, X., Ren, J., Qu, K., and Qu, X. (2012). Using graphene oxide high nearinfrared absorbance for photothermal treatment of alzheimer's disease. $A d v$. Mater. 24, 1722-1728. doi: 10.1002/adma.201104864

Ling, X., Lee, Y.-H., Lin, Y., Fang, W., Yu, L., Dresselhaus, M. S., et al. (2014). Role of the seeding promoter in MoS2 growth by chemical vapor deposition. Nano Lett. 14, 464-472. doi: 10.1021/nl4033704

Liu, C., Kong, D., Hsu, P.-C., Yuan, H., Lee, H.-W., Liu, Y., et al. (2016). Rapid water disinfection using vertically aligned MoS2 nanofilms and visible light. Nat. Nanotechnol. 11, 1098-1104. doi: 10.1038/nnano.2016.138

Liu, K.-K., Zhang, W., Lee, Y.-H., Lin, Y.-C., Chang, M.-T., Su, C.-Y., et al. (2012). Growth of large-area and highly crystalline MoS2 thin layers on insulating substrates. Nano Lett. 12, 1538-1544. doi: 10.1021/nl2043612

Liu, Y., Ai, K., and Lu, L. (2012a). Nanoparticulate X-ray computed tomography contrast agents: from design validation to in vivo applications. Acc. Chem. Res. 45, 1817-1827. doi: 10.1021/ar300150c

Liu, Y., Dong, X., and Chen, P. (2012b). Biological and chemical sensors based on graphene materials. Chem. Soc. Rev. 41, 2283-2307. doi: 10.1039/C1CS15270J

Liu, Z., Li, Z., Liu, J., Gu, S., Yuan, Q., Ren, J., et al. (2012). Long-circulating Er3+doped $\mathrm{Yb} 2 \mathrm{O} 3$ up-conversion nanoparticle as an in vivo X-Ray CT imaging contrast agent. Biomaterials 33, 6748-6757. doi: 10.1016/j.biomaterials.2012. 06.033

Liu, S., Shen, Z., Wu, B., Yu, Y., Hou, H., Zhang, X.-X., et al. (2017). Cytotoxicity and efflux pump inhibition induced by molybdenum disulfide and boron nitride nanomaterials with sheetlike structure. Environ. Sci. Technol. 51, 10834-10842. doi: 10.1021/acs.est.7b02463

Liu, T., and Liu, Z. (2018). 2D MoS2 nanostructures for biomedical applications. Adv. Healthc. Mater. 7:1701158. doi: 10.1002/adhm.201701158

Liu, T., Wang, C., Cui, W., Gong, H., Liang, C., Shi, X., et al. (2014a). Combined photothermal and photodynamic therapy delivered by PEGylated MoS2 nanosheets. Nanoscale 6, 11219-11225. doi: 10.1039/C4NR03753G

Liu, T., Wang, C., Gu, X., Gong, H., Cheng, L., Shi, X., et al. (2014b). Drug delivery with PEGylated MoS2 nano-sheets for combined photothermal and chemotherapy of cancer. Adv. Mater. 26, 3433-3440. doi: 10.1002/adma. 201305256

Liu, W., Zhang, X., Zhou, L., Shang, L., and Su, Z. (2019). Reduced graphene oxide (rGO) hybridized hydrogel as a near-infrared (NIR)/pH dual-responsive platform for combined chemo-photothermal therapy. J. Colloid Interf. Sci. 536, 160-170. doi: 10.1016/j.jcis.2018.10.050

Liu, X., Miller, A. L., Park, S., George, M. N., Waletzki, B. E., Xu, H., et al. (2019). Two-dimensional black phosphorus and graphene oxide nanosheets synergistically enhance cell proliferation and osteogenesis on $3 \mathrm{D}$ printed scaffolds. ACS Appl. Mater. Inter. 11, 23558-23572. doi: 10.1021/acsami. $9 \mathrm{~b} 04121$

Lu, C., Liu, Y., Ying, Y., and Liu, J. (2017). Comparison of MoS2, WS2, and graphene oxide for DNA adsorption and sensing. Langmuir 33, 630-637. doi: 10.1021/acs.langmuir.6b04502

Lukowski, M. A., Daniel, A. S., Meng, F., Forticaux, A., Li, L., and Jin, S. (2013). Enhanced hydrogen evolution catalysis from chemically exfoliated metallic MoS2 nanosheets. J. Am. Chem. Soc. 135, 10274-10277. doi: 10.1021/ja404523s

Ma, Y.-H., Dou, W.-T., Pan, Y.-F., Dong, L.-W., Tan, Y.-X., He, X.-P., et al. (2017). Fluorogenic 2D peptidosheet unravels CD47 as a potential biomarker for profiling hepatocellular carcinoma and cholangiocarcinoma tissues. Adv. Mater. 29:1604253. doi: 10.1002/adma.201604253

Novoselov, K. S., Geim, A. K., Morozov, S. V., Jiang, D., Zhang, Y., Dubonos, S. V., et al. (2004). Electric field effect in atomically thin carbon films. Science 306, 666-669. doi: 10.1126/science.1102896

Oudeng, G., Au, M., Shi, J., Wen, C., and Yang, M. (2018). One-step in situ detection of miRNA-21 expression in single cancer cells based on biofunctionalized MoS2 nanosheets. ACS Appl. Mater. Inter. 10, 350-360. doi: 10.1021/acsami.7b18102

Pak, J., Jang, Y., Byun, J., Cho, K., Kim, T.-Y., Kim, J.-K., et al. (2018). Twodimensional thickness-dependent avalanche breakdown phenomena in MoS2 
field-effect transistors under high electric fields. ACS Nano 12, 7109-7116. doi: 10.1021/acsnano.8b02925

Park, Y., Ryu, B., Oh, B.-R., Song, Y., Liang, X., and Kurabayashi, K. (2017). Biotunable nanoplasmonic filter on few-layer MoS2 for rapid and highly sensitive cytokine optoelectronic immunosensing. ACS Nano 11, 5697-5705. doi: 10.1021/acsnano.7b01162

Pelin, M., Fusco, L., Martín, C., Sosa, S., Frontiñán-Rubio, J., GonzálezDomínguez, J. M., et al. (2018). Graphene and graphene oxide induce ROS production in human $\mathrm{HaCaT}$ skin keratinocytes: the role of xanthine oxidase and NADH dehydrogenase. Nanoscale 10, 11820-11830. doi: 10.1039/ C8NR02933D

Peng, Y., Meng, Z., Zhong, C., Lu, J., Yu, W., Jia, Y., et al. (2001a). Hydrothermal synthesis and characterization of single-molecular-layer MoS2 and MoSe2. Chem. Lett. 30, 772-773. doi: 10.1246/cl.2001.772

Peng, Y., Meng, Z., Zhong, C., Lu, J., Yu, W., Yang, Z., et al. (2001b). Hydrothermal synthesis of MoS2 and its pressure-related crystallization. J. Solid State Chem. 159, 170-173. doi: 10.1006/jssc.2001.9146

Presolski, S., Wang, L., Loo, A. H., Ambrosi, A., Lazar, P., Ranc, V., et al. (2017). Functional nanosheet synthons by covalent modification of transition-metal dichalcogenides. Chem. Mater. 29, 2066-2073. doi: 10.1021/acs.chemmater. $6 \mathrm{~b} 04171$

Qian, X., Shen, S., Liu, T., Cheng, L., and Liu, Z. (2015). Two-dimensional TiS2 nanosheets for in vivo photoacoustic imaging and photothermal cancer therapy. Nanoscale 7, 6380-6387. doi: 10.1039/C5NR00893J

Qin, Y., Zhou, Z.-W., Pan, S.-T., He, Z.-X., Zhang, X., Qiu, J.-X., et al. (2015). Graphene quantum dots induce apoptosis, autophagy, and inflammatory response via p38 mitogen-activated protein kinase and nuclear factor- $\mathrm{KB}$ mediated signaling pathways in activated THP-1 macrophages. Toxicology 327 , 62-76. doi: 10.1016/j.tox.2014.10.011

Radisavljevic, B., Radenovic, A., Brivio, J., Giacometti, V., and Kis, A. (2011). Single-layer MoS2 transistors. Nat. Nanotechnol. 6, 147-150. doi: 10.1038/ nnano.2010.279

Ramakrishna Matte, H. S. S., Gomathi, A., Manna, A. K., Late, D. J., Datta, R., Pati, S. K., et al. (2010). MoS2 and WS2 analogues of graphene. Angew. Chem. Int. Ed. 49, 4059-4062. doi: 10.1002/anie.201000009

Roy, S., Sarkar, A., and Jaiswal, A. (2019). Poly(allylamine hydrochloride)functionalized reduced graphene oxide for synergistic chemo-photothermal therapy. Nanomedicine 14, 255-274. doi: 10.2217/nnm-2018-0320

Shamberger, R. J. (1981). Selenium in the environment. Sci. Total Environ. 17, 59-74. doi: 10.1016/0048-9697(81)90108-X

Shen, J., Wu, J., Wang, M., Ge, Y., Dong, P., Baines, R., et al. (2016). Insight into in situ amphiphilic functionalization of few-layered transition metal dichalcogenide nanosheets. Adv. Mater. 28, 8469-8476. doi: 10.1002/adma. 201602887

Shi, L., Chen, J., Teng, L., Wang, L., Zhu, G., Liu, S., et al. (2016). The antibacterial applications of graphene and its derivatives. Small 12, 4165-4184. doi: 10.1002/ smll.201601841

Shibu, E. S., Hamada, M., Murase, N., and Biju, V. (2013). Nanomaterials formulations for photothermal and photodynamic therapy of cancer. J. Photochem. Photobiol. C Photochem. Rev. 15, 53-72. doi: 10.1016/j. jphotochemrev.2012.09.004

Shilo, M., Reuveni, T., Motiei, M., and Popovtzer, R. (2012). Nanoparticles as computed tomography contrast agents: current status and future perspectives. Nanomedicine 7, 257-269. doi: 10.2217/nnm.11.190

Si, M., Su, C.-J., Jiang, C., Conrad, N. J., Zhou, H., Maize, K. D., et al. (2018). Steepslope hysteresis-free negative capacitance MoS2 transistors. Nat. Nanotechnol. 13, 24-28. doi: 10.1038/s41565-017-0010-1

Splendiani, A., Sun, L., Zhang, Y., Li, T., Kim, J., Chim, C.-Y., et al. (2010). Emerging photoluminescence in monolayer MoS2. Nano Lett. 10, 1271-1275. doi: $10.1021 / \mathrm{nl} 903868 \mathrm{w}$

Suhito, I. R., Han, Y., Kim, D.-S., Son, H., and Kim, T.-H. (2017). Effects of two-dimensional materials on human mesenchymal stem cell behaviors. Biochem. Biophys. Res. Commun. 493, 578-584. doi: 10.1016/j.bbrc.2017. 08.149

Teo, W. Z., Chng, E. L. K., Sofer, Z., and Pumera, M. (2014). Cytotoxicity of exfoliated transition-metal dichalcogenides (MoS2, WS2, and WSe2) is lower than that of graphene and its analogues. Chem. Eur. J. 20, 9627-9632. doi: $10.1002 /$ chem. 201402680
Vega-Mayoral, V., Backes, C., Hanlon, D., Khan, U., Gholamvand, Z., O’brien, M., et al. (2016). Photoluminescence from liquid-exfoliated WS2 monomers in poly(vinyl alcohol) polymer composites. Adv. Funct. Mater. 26, 1028-1039. doi: $10.1002 /$ adfm. 201503863

Wang, J., Yan, M., Zhao, K., Liao, X., Wang, P., Pan, X., et al. (2017). Field effect enhanced hydrogen evolution reaction of MoS2 nanosheets. Adv. Mater. 29:1604464. doi: 10.1002/adma.201604464

Wang, T., Chen, S., Pang, H., Xue, H., and Yu, Y. (2017). MoS2-based nanocomposites for electrochemical energy storage. Adv. Sci. 4:1600289. doi: 10.1002/advs.201600289

Wang, L. V., and Hu, S. (2012). Photoacoustic tomography:in vivo imaging from organelles to organs. Science 335, 1458-1462. doi: 10.1126/science.1216210

Wang, S., Chen, Y., Li, X., Gao, W., Zhang, L., Liu, J., et al. (2015a). Injectable 2D MoS2-integrated drug delivering implant for highly efficient NIR-triggered synergistic tumor hyperthermia. Adv. Mater. 27, 7117-7122. doi: 10.1002/adma. 201503869

Wang, S., Li, K., Chen, Y., Chen, H., Ma, M., Feng, J., et al. (2015b). Biocompatible PEGylated MoS2 nanosheets: Controllable bottom-up synthesis and highly efficient photothermal regression of tumor. Biomaterials 39, 206-217. doi: 10. 1016/j.biomaterials.2014.11.009

Wang, S., Rong, Y., Fan, Y., Pacios, M., Bhaskaran, H., He, K., et al. (2014). Shape evolution of monolayer MoS2 crystals grown by chemical vapor deposition. Chem. Mater. 26, 6371-6379. doi: 10.1021/cm5025662

Wang, X., Feng, H., Wu, Y., and Jiao, L. (2013). Controlled synthesis of highly crystalline MoS2 flakes by chemical vapor deposition. J. Am. Chem. Soc. 135, 5304-5307. doi: 10.1021/ja4013485

Xi, Q., Zhou, D.-M., Kan, Y.-Y., Ge, J., Wu, Z.-K., Yu, R.-Q., et al. (2014). Highly sensitive and selective strategy for MicroRNA detection based on WS2 nanosheet mediated fluorescence quenching and duplex-specific nuclease signal amplification. Anal. Chem. 86, 1361-1365. doi: 10.1021/ac403944c

Yang, B., Chen, Y., and Shi, J. (2018). Material chemistry of two-dimensional inorganic nanosheets in cancer theranostics. Chem 4, 1284-1313. doi: 10.1016/ j.chempr.2018.02.012

Yang, X., Li, J., Liang, T., Ma, C., Zhang, Y., Chen, H., et al. (2014). Antibacterial activity of two-dimensional MoS2 sheets. Nanoscale 6, 10126-10133. doi: 10. 1039/C4NR01965B

Yin, W., Yan, L., Yu, J., Tian, G., Zhou, L., Zheng, X., et al. (2014). High-throughput synthesis of single-layer MoS2 nanosheets as a near-infrared photothermaltriggered drug delivery for effective cancer therapy. ACS Nano 8, 6922-6933. doi: $10.1021 / \mathrm{nn} 501647 \mathrm{j}$

Yong, Y., Zhou, L., Gu, Z., Yan, L., Tian, G., Zheng, X., et al. (2014). WS2 nanosheet as a new photosensitizer carrier for combined photodynamic and photothermal therapy of cancer cells. Nanoscale 6, 10394-10403. doi: 10.1039/C4NR02453B

Yu, J., Yin, W., Zheng, X., Tian, G., Zhang, X., Bao, T., et al. (2015). Smart $\mathrm{MoS} 2 / \mathrm{Fe} 3 \mathrm{O} 4$ nanotheranostic for magnetically targeted photothermal therapy guided by magnetic resonance/photoacoustic imaging. Theranostics 5, 931-945. doi: 10.7150/thno.11802

Yu, P., Fu, W., Zeng, Q., Lin, J., Yan, C., Lai, Z., et al. (2017). Controllable synthesis of atomically thin type-II weyl semimetal WTe2 nanosheets: an advanced electrode material for all-solid-state flexible supercapacitors. $A d v$. Mater. 29:1701909. doi: 10.1002/adma.201701909

Yu, Y., Wu, N., Yi, Y., Li, Y., Zhang, L., Yang, Q., et al. (2017). Dispersible MoS2 nanosheets activated TGF- $\beta /$ Smad pathway and perturbed the metabolome of human dermal fibroblasts. ACS Biomater. Sci. Eng. 3, 3261-3272. doi: 10.1021/ acsbiomaterials.7b00575

Yuan, P., Zhou, Q., and Hu, X. (2018). The phases of WS2 nanosheets influence uptake, oxidative stress, lipid peroxidation, membrane damage, and metabolism in algae. Environ. Sci. Technol. 52, 13543-13552. doi: 10.1021/acs.est.8b04444

Yuan, Y., Li, R., and Liu, Z. (2014). Establishing water-soluble layered WS2 nanosheet as a platform for biosensing. Anal. Chem. 86, 3610-3615. doi: 10. $1021 /$ ac5002096

Zeng, Z., Yin, Z., Huang, X., Li, H., He, Q., Lu, G., et al. (2011). Single-layer semiconducting nanosheets: high-yield preparation and device fabrication. Angew. Chem. Int. Ed. 50, 11093-11097. doi: 10.1002/anie.201106004

Zhang, M., Cao, Y., Chong, Y., Ma, Y., Zhang, H., Deng, Z., et al. (2013). Graphene oxide based theranostic platform for T1-weighted magnetic resonance imaging and drug delivery. ACS Appl. Mater. Inter. 5, 13325-13332. doi: 10.1021/ am404292e 
Zhang, M., Zhou, N., Yuan, P., Su, Y., Shao, M., and Chi, C. (2017). Graphene oxide and adenosine triphosphate as a source for functionalized carbon dots with applications in $\mathrm{pH}$-triggered drug delivery and cell imaging. $R S C A d v .7$, 9284-9293. doi: 10.1039/C6RA27887F

Zhang, Y., Zhang, H., Wang, Y., Wu, H., Zeng, B., Zhang, Y., et al. (2017). Hydrophilic graphene oxide/bismuth selenide nanocomposites for CT imaging, photoacoustic imaging, and photothermal therapy. J. Mat. Chem. B 5, 1846-1855. doi: 10.1039/C6TB02137A

Zhao, J., Wei, Z., Zhang, Q., Yu, H., Wang, S., Yang, X., et al. (2019). Static and dynamic piezopotential modulation in piezo-electret gated MoS2 field-effect transistor. ACS Nano 13, 582-590. doi: 10.1021/acsnano.8b07477

Zhou, X., Jia, J., Luo, Z., Su, G., Yue, T., and Yan, B. (2019). Remote induction of cell autophagy by 2D MoS2 nanosheets via perturbing cell surface receptors and mTOR pathway from outside of cells. ACS Appl. Mater. Inter. 11, 6829-6839. doi: 10.1021/acsami.8b21886

Zhou, X., and Yan, B. (2019). Induction of mTOR-dependent autophagy by WS2 nanosheets from both inside and outside of human cells. Nanoscale 11, 10684-10694. doi: 10.1039/C9NR02850A
Zhu, C., Zeng, Z., Li, H., Li, F., Fan, C., and Zhang, H. (2013). Single-layer MoS2based nanoprobes for homogeneous detection of biomolecules. J. Am. Chem. Soc. 135, 5998-6001. doi: 10.1021/ja4019572

Zou, W., Zhang, X., Zhao, M., Zhou, Q., and Hu, X. (2017). Cellular proliferation and differentiation induced by single-layer molybdenum disulfide and mediation mechanisms of proteins via the Akt-mTOR-p70S6K signaling pathway. Nanotoxicology 11, 781-793. doi: 10.1080/17435390.2017.1357213

Conflict of Interest: The authors declare that the research was conducted in the absence of any commercial or financial relationships that could be construed as a potential conflict of interest.

Copyright (c) 2020 Zhou, Sun and Bai. This is an open-access article distributed under the terms of the Creative Commons Attribution License (CC BY). The use, distribution or reproduction in other forums is permitted, provided the original author(s) and the copyright owner(s) are credited and that the original publication in this journal is cited, in accordance with accepted academic practice. No use, distribution or reproduction is permitted which does not comply with these terms. 